\title{
El efecto sustitución y la evolución del costo de vida en la frontera de Uruguay con Brasil
}

\author{
Fernando Correa Alsina*
}

Resumen. El consumidor que vive próximo a la frontera de Uruguay con Brasil tiene la posibilidad de comprar bienes en ambas márgenes de la misma. Puede sustituir compras de un país hacia el otro según la evolución relativa de los precios. De esta manera, tiene acceso a un menor costo de vida que un consumidor ubicado en el interior del país. Además, si el tipo de cambio real bilateral experimenta fluctuaciones importantes, puede sacar un provecho significativo de su localización. Este trabajo aborda el tema desde un punto de vista teórico y empírico. Por un lado, se presenta una adaptación de la teoría de los índices del costo de vida a la situación planteada. Por otro, las estimaciones realizadas muestran que la ventaja derivada de vivir en la frontera fue muy significativa en la última década.

Palabras clave: frontera, consumo, costo, gasto, alimentos.

\begin{abstract}
The consumers that live near the border Uruguay-Brazil have the possibility of buying goods on both sides of such border. They may replace purchases of one country to the other according to the evolution of prices. In this way, consumers have a lower cost of living compared to the consumers living in the rest of the country. Besides, if the real bilateral exchange rate undergoes important fluctuations, they may take significant advantage of their location. This paper deals with the issue from a theoretical and empirical point of view. On one hand, it presents an adaptation of the cost-of-living index theory to the situation, and on the other hand, the estimates show that the advantages of living on the border were really significant in the last decade.

Keywords: border, consume, cost, expense, food supplies.
\end{abstract}

*Docente de la Facultad de Ciencias Sociales, Universidad de la República-Regional Norte-Salto, Montevideo, Uruguay. Correo electrónico: fcorrea@aladi.org 


\section{Introducción}

El consumidor que vive próximo a la frontera de un país tiene la posibilidad de comprar bienes y servicios en ambas márgenes de la misma. De esta forma puede sustituir compras realizadas en un país hacia el otro según la evolución relativa de los precios.

En la medida en que el consumidor fronterizo dispone de esta posibilidad adicional de sustitución, que otros consumidores ubicados en el interior del país no tienen, puede tener acceso a un menor costo de vida. Si además el tipo de cambio real bilateral entre los dos países fronterizos experimenta fluctuaciones importantes, en determinadas situaciones podrá sacar un provecho significativo de la sustitución de bienes entre ambas márgenes.

El presente trabajo intenta abordar el tema planteado tanto desde un punto de vista teórico como empírico. Por un lado, se realiza una adaptación de la teoría de los índices del costo de vida a la situación planteada. Por otro, se realizan estimaciones de la evolución del costo de vida en alimentos y bebidas durante el periodo 1995-2005 para consumidores representativos que viven en tres lugares diferentes: la zona fronteriza del Uruguay con Brasil, en el interior del país, y en la capital. A partir de estas estimaciones se calcula la ventaja de vivir en la frontera.

El trabajo se organiza así: en la primera sección se esboza una introducción al fenómeno de estudio, en la segunda se da una dimensión de la importancia del tema en Uruguay; en la tercera se analiza la evidencia disponible a partir de la Encuesta de Gastos e Ingresos de los Hogares 1994-1995; en la cuarta sección se presenta un marco teórico y un índice de evolución del costo de vida que permite abordar el tema en un periodo más amplio (1995-2005); en la quinta sección se describen los diferentes datos utilizados para la estimación; en la sexta se analizan los resultados de las estimaciones realizadas para dicho periodo; y finalmente, se resumen las conclusiones.

\section{Relevancia del tema}

La particular ubicación geográfica del Uruguay ha determinado que tenga una amplia frontera con sus dos países vecinos. Con la 
Argentina se encuentra separado por el Río Uruguay y sobre esta frontera se encuentran tres capitales departamentales: Salto, Paysandú y Fray Bentos.

Por su parte, la frontera con el Brasil está delimitada en algunos tramos por corrientes de agua: los ríos Cuareim y Yaguarón, y la laguna Merín. El resto es "frontera seca". Sobre la misma se ubican las dos capitales departamentales más lejanas de la capital: Artigas y Rivera. Allí también están otras ciudades de menor tamaño relativo como Bella Unión, Río Branco y Chuy.

Favorecidos por la geografía, los uruguayos han desarrollado la costumbre bastante extendida, de realizar compras en los países vecinos, sobre todo en el Brasil. Esta costumbre, conocida en la jerga popular con el nombre de bagayear, no abarca solamente a los habitantes de las ciudades fronterizas. Se extiende a poblados interiores próximos a la frontera, llegando a otras capitales departamentales como Melo y Tacuarembó, así como a los veraneantes que se encuentran en las playas del extremo este del país durante la época de festival.

Figura 1

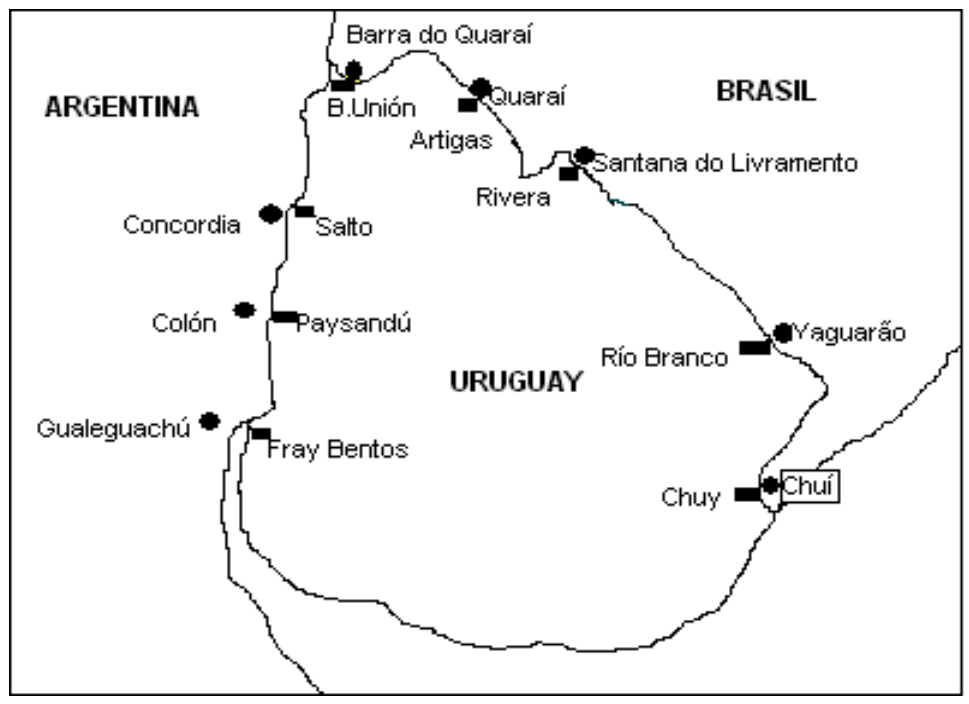

Fuente: Elaboración propia. 
Las dos fronteras mencionadas tienen características diferentes. Las cinco ciudades ubicadas sobre la frontera con el Brasil se encuentran a corta distancia, o unidas, a centros poblados brasileños. Es así que Artigas se encuentra frente a Quaraí, Bella Unión a Barra do Quaraí, Rivera a Santana do Livramento, Río Branco a Yaguarão y Chuy a Chuí.

Por el contrario, las ciudades que se ubican sobre la frontera con la Argentina se encuentran a una mayor distancia de los centros poblados del vecino país. Por ejemplo, Salto, Paysandú y Fray Bentos están a más de 10 kilómetros por vía terrestre ${ }^{1}$ de Concordia, Colón y Gualeguaychú, respectivamente.

Lo anterior ha determinado que la costumbre de bagayear sea más propia de los habitantes de las ciudades fronterizas con Brasil.

Según los datos del censo del año 2004, 5.2\% de la población uruguaya es fronteriza, definiendo como tal aquella que habita a menos de 10 kilómetros de algún poblado de un país vecino (cuadro 1). Su participación es significativa en determinados departamentos. En particular en Artigas y Rivera supera el 70\% del total, mientras que en Cerro Largo y Rocha ronda el 20 por ciento.

Sin perjuicio de la población involucrada, el tema carecería de relevancia si no fuera por las bruscas fluctuaciones que experimentó el tipo de cambio real entre Uruguay y Brasil en el pasado reciente. Esto ha determinado que el consumidor de la zona se haya volcado a realizar sus compras a un lado y otro de la frontera según la conveniencia.

En particular, analizando la evolución del tipo de cambio real bilateral 1995-2005 es posible distinguir cuatro periodos (gráfica 1). El primero, marcado por una relativa estabilidad, abarca desde 1995 hasta la devaluación de la moneda brasileña ocurrida en enero de 1999. El segundo, caracterizado por el bajo nivel del tipo de cambio real, se inicia con la devaluación y se extiende hasta julio de 2002. El tercero, pautado por la recuperación, comienza con la devaluación uruguaya y se prolonga hasta marzo de 2003; finalmente, desde allí

\footnotetext{
${ }^{1}$ Salto se encuentra ubicada frente a la ciudad argentina de Concordia. Sin embargo, el puente internacional se ubica más al norte de ambas ciudades, lo que determina que la distancia terrestre entre ambas sea de aproximadamente 40 kilómetros.
} 


\section{Cuadro 1}

Población fronteriza del Uruguaya

Año 2004

\begin{tabular}{|c|c|c|c|}
\hline Localidad & Población & $\%$ del dpto. & \% del país \\
\hline Artigas & 41,687 & 53.4 & \\
\hline Bella Unión & 13,187 & 16.9 & \\
\hline Las Piedras & 2,164 & 2.8 & \\
\hline Pintadito & 1,487 & 1.9 & \\
\hline Franquia & 833 & 1.1 & \\
\hline Cuareim & 780 & 1.0 & \\
\hline Cerro Ejido & 516 & 0.7 & \\
\hline Coronado & 469 & 0.6 & \\
\hline Port. de Hierro y Campodónico & 404 & 0.5 & \\
\hline Cerro Signorelli & 192 & 0.2 & \\
\hline Cerro San Eugenio & 95 & 0.1 & \\
\hline Subtotal dpto. de Artigas & 61,814 & 79.2 & 1.9 \\
\hline Rivera & 64,426 & 61.4 & \\
\hline Mandubí & 5,157 & 4.9 & \\
\hline La Pedrera & 2,887 & 2.8 & \\
\hline Santa Teresa & 2,171 & 2.1 & \\
\hline Lagunón & 2,154 & 2.1 & \\
\hline Lagos del Norte & 178 & 0.2 & \\
\hline Subtotal dpto. de Rivera & 76,973 & 73.4 & 2.4 \\
\hline Río Branco & 13,456 & 15.5 & \\
\hline Isidoro Noblía & 2,462 & 2.8 & \\
\hline Aceguá & 1,493 & 1.7 & \\
\hline Subtotal dpto. de Cerro Largo & 17,411 & 20.1 & 0.5 \\
\hline Chuy & 10,401 & 14.9 & \\
\hline 18 de Julio & 1,191 & 1.7 & \\
\hline Puimayen & 503 & 0.7 & \\
\hline Barra del Chuy & 367 & 0.5 & \\
\hline Subtotal dpto. de Rocha & 12,462 & 17.8 & 0.4 \\
\hline Total & 168,660 & & 5.2 \\
\hline
\end{tabular}

Fuente: CENSO 2004.

a Se consideró fronteriza a la población que se encuentra a menos de $10 \mathrm{~km}$ de un poblado ubicado en la otra margen de la frontera, utilizando un medio de transporte terrestre. 
Gráfica 1

Tipo de cambio real Uruguay-Brasil

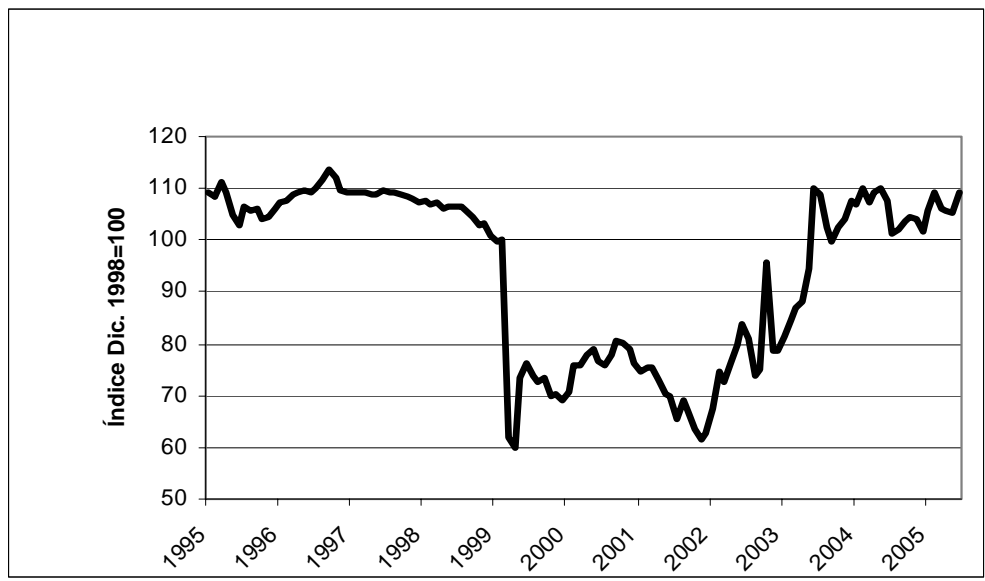

hasta la actualidad se mantuvo en un nivel similar al existente con anterioridad a 1999.

Evidencia surgida de la Encuesta de Gastos e Ingresos de los Hogares 1994-1995

Si bien el fenómeno del consumo transfronterizo de bienes y servicios en la frontera uruguayo-brasileña es bien conocido en el país, no existe evidencia empírica sobre la incidencia que tiene en el costo de vida de la población. Esto se debe, en parte, a que no existe en Uruguay un relevamiento periódico de precios al consumo en el interior del país que permita realizar una comparación con los de Brasil. El índice de precios al consumo (IPC) se elabora únicamente con precios de Montevideo.

Sin perjuicio de lo anterior, la encuesta que se elabora, aproximadamente cada 12 años, para actualizar la canasta con que se calcula el IPC se extiende a algunas ciudades del interior del país. Concretamente la última, denominada Encuesta de Gastos e Ingresos de los 
Hogares 1994-1995 (EGIH 1994-1995) y relevada en el periodo que va desde junio de 1994 a mayo de 1995, comprendió además de Montevideo a cinco ciudades del interior del país: Colonia, Maldonado, Durazno, Salto y Rivera. Esta última es la única ciudad cuya población es fronteriza según la definición adoptada anteriormente.

La EGIH 1994-1995 aporta evidencia sobre el efecto que tuvo, en ese periodo concreto, el consumo transfronterizo en el costo de vida de los habitantes de Rivera en comparación con los de las otras ciudades encuestadas. Sin embargo, si bien la EGIH 1994-1995 se extiende a todos los rubros del consumo, solamente la información correspondiente a alimentos y bebidas cuenta con medición de las cantidades consumidas y permite, como veremos, comparar el costo de vida.

Una primera aproximación consiste en comparar el gasto promedio mensual, a precios de noviembre de 1994, en alimentos y bebidas realizado por los hogares de las seis ciudades encuestadas. De dicha comparación surge claramente que el hogar promedio de Rivera es el que tiene un menor gasto en alimentos y bebidas (cuadro 2).

No obstante, esto no permite concluir que Rivera sea la ciudad con menor costo de vida en estos rubros, por varias razones. Una de

\section{Cuadro 2}

Gasto en alimentos y bebidas según ciudades a precios de noviembre de 1994

\begin{tabular}{lcc} 
Ciudad & \multicolumn{2}{c}{ Gasto mensual en alimentos y bebidas } \\
\cline { 2 - 3 } & Por hogar & Per cápita \\
\hline Montevideo & 1,741 & 549 \\
Colonia & 1,356 & 436 \\
Durazno & 1,214 & 342 \\
Maldonado & 1,678 & 528 \\
Rivera & $\mathbf{1 , 1 7 0}$ & $\mathbf{3 3 1}$ \\
Salto & 1,319 & 353 \\
\hline
\end{tabular}

Fuente: EGIH 1994-1995.

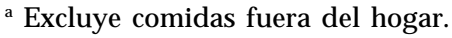


ellas es el hecho de que los hogares tienen un diferente número de integrantes según ciudad, lo que explicaría, al menos en parte, las diferencias presentadas en el gasto. Sin embargo, si para corregir este efecto comparamos el gasto per cápita promedio en alimentos y bebidas de las diferentes ciudades, vemos que el ordenamiento original se mantiene. De mayor a menor gasto se ubican: Montevideo, Maldonado, Colonia, Salto, Durazno y Rivera.

Esta segunda comparación sigue adoleciendo de un problema a los efectos de extraer conclusiones sobre el costo de vida relativo en las diferentes ciudades. Las diferencias de gasto pueden explicarse por diferencias en las cantidades consumidas y no solamente en los precios.

Para superar esta limitante, se procedió a calcular el gasto promedio que tendrían los hogares de las diferentes ciudades si consumieran una canasta homogénea avaluada a los precios de cada localidad. Como canasta homogénea, y para efectos de analizar la solidez de los resultados, se consideraron las correspondientes a cada una de las ciudades consideradas.

Los precios obtenidos para cada ciudad surgen de dividir el gasto realizado en cada artículo sobre las cantidades consumidas del mismo. Por esto son, técnicamente hablando, valores unitarios y no precios. ${ }^{2}$

Los resultados muestran que, cualquiera sea la canasta considerada a los efectos del cálculo, Rivera es la ciudad con el menor costo de vida (cuadro 3). El ordenamiento es prácticamente siempre el mismo, de mayor a menor: Montevideo, Maldonado, Colonia, Durazno, Salto y Rivera. ${ }^{3}$

Si bien el ordenamiento es robusto, la escasa significación de las diferencias de costo de vida en algunos casos permite hablar de que existen tres niveles de costo de vida: Montevideo en un extremo, las ciudades del interior no fronterizas en un plano intermedio y Rivera en el otro.

El costo de vida en Montevideo es un 15\% superior al correspondiente al interior no fronterizo, y éste a su vez se encuentra $24 \%$ por encima del correspondiente a Rivera.

\footnotetext{
${ }^{2}$ El uso de valores unitarios implica suponer que los bienes consumidos en las diferentes ciudades son homogéneos.

${ }^{3}$ Este ordenamiento solamente cambia cuando se considera la canasta de Durazno, en cuyo caso esta ciudad pasa a tener un menor costo de vida que Salto.
} 


\section{Cuadro 3}

Gasto en alimentos y bebidas según ciudades, con canasta homogénea a precios de noviembre de 1994 de cada departamento ${ }^{a}$

\begin{tabular}{lllllll}
\hline \multirow{2}{*}{ Ciudad } & \multicolumn{6}{c}{ Canasta } \\
\cline { 2 - 7 } & Montevideo Colonia & Durazno & Maldonado & Rivera & Salto \\
\hline Montevideo & 1,741 & 1,694 & 1,433 & 1,845 & 1,707 & 1,687 \\
Colonia & 1,615 & 1,356 & 1,275 & 1,642 & 1,534 & 1,405 \\
Durazno & 1,581 & 1,347 & 1,214 & 1,607 & 1,501 & 1,354 \\
Maldonado & 1,715 & 1,487 & 1,370 & 1,678 & 1,587 & 1,481 \\
Rivera & $\mathbf{1 , 3 1 3}$ & $\mathbf{1 , 1 3 2}$ & $\mathbf{1 , 0 5 4}$ & $\mathbf{1 , 3 1 9}$ & $\mathbf{1 , 1 7 0}$ & $\mathbf{1 , 1 4 2}$ \\
Salto & 1,570 & 1,345 & 1,241 & 1,580 & 1,455 & 1,319 \\
\hline
\end{tabular}

Fuente: EGIH 1994-1995.

a Excluye comidas fuera del hogar.

Entre Montevideo y el interior urbano existen diferencias en el tamaño de las ciudades y en las realidades socioeconómicas que pueden explicar el superior costo de vida registrado en la capital. Sin embargo, la significativa diferencia entre Rivera y el resto del interior urbano sólo puede atribuirse, en un país pequeño e integrado económicamente, al "efecto frontera".

Los hogares relevados por la EGIH 1994-1995 son interrogados acerca de las cantidades consumidas y el gasto realizado. En el caso de Rivera, este último incluye tanto el realizado en el territorio uruguayo como en la margen brasileña. ${ }^{4}$ De esta manera, si el consumidor adquirió los bienes en el país más barato, obtuvo un precio inferior, o igual, al registrado en la margen uruguaya, y realizó, por tanto, un menor gasto que el habitante del interior del país.

${ }^{4}$ La propia EGIH 1994-1995 previó una pregunta en el cuestionario que apuntaba a determinar si el gasto era realizado en plaza o en el exterior. A pesar de ello, el Instituto Nacional de Estadística no incorporó la información correspondiente a la variable aludida en los resultados que presenta. 


\section{Marco teórico}

A los efectos de extender la medición sobre la evolución del costo de vida al periodo 1995-2005, es necesario definir previamente un marco conceptual. Concretamente, es preciso determinar cuál es el índice de costo de vida de un consumidor que vive en la frontera del país y que puede no solamente sustituir bienes en su canasta, sino también sustituir el país donde los compra.

\section{La teoría sobre los índices de costo de vida}

La mayoría de los países utilizan índices del tipo Laspeyres para medir la evolución de los precios y en particular la inflación. Dichos índices, como se sabe, tienen ponderaciones fijas por lo que no dan cuenta de la sustitución que realizan los consumidores entre diferentes bienes, entre diversas fuentes (supermercados, almacenes, ferias, panaderías, carnicerías, etc.) y entre zonas (barrios). Por esta razón no se adaptan a la situación que se desea medir en el presente trabajo.

Existe evidencia empírica que demuestra que los índices de precios al consumo (IPC) en la mayoría de los países tienden a sobreestimar la verdadera evolución del costo de vida (Moulton, 1996; Shapiro y Wilcox, 1997). ${ }^{5}$ Esto se debe, en parte, a que los mismos no dan cuenta del efecto sustitución.

Esto puede observarse en la gráfica 2, donde se representa la situación de un consumidor que adquiere dos bienes. Inicialmente, se encuentra en equilibrio en el punto A alcanzando un determinado nivel de utilidad representado por la curva $\mathrm{U}$ y con un determinado costo C. Posteriormente, el bien 2 se encarece en términos relativos al bien 1, lo que cambia la pendiente de la recta de restricción presupuestaria. Si el consumidor continúa comprando las mismas canti-

\footnotetext{
${ }^{5}$ Esto se debe no solamente a las razones antes mencionadas, sino también a que no recogen adecuadamente otros fenómenos como los cambios de calidad de los bienes y la aparición de artículos nuevos.
} 
Gráfica 2

Evolución del costo con y sin efecto sustitución

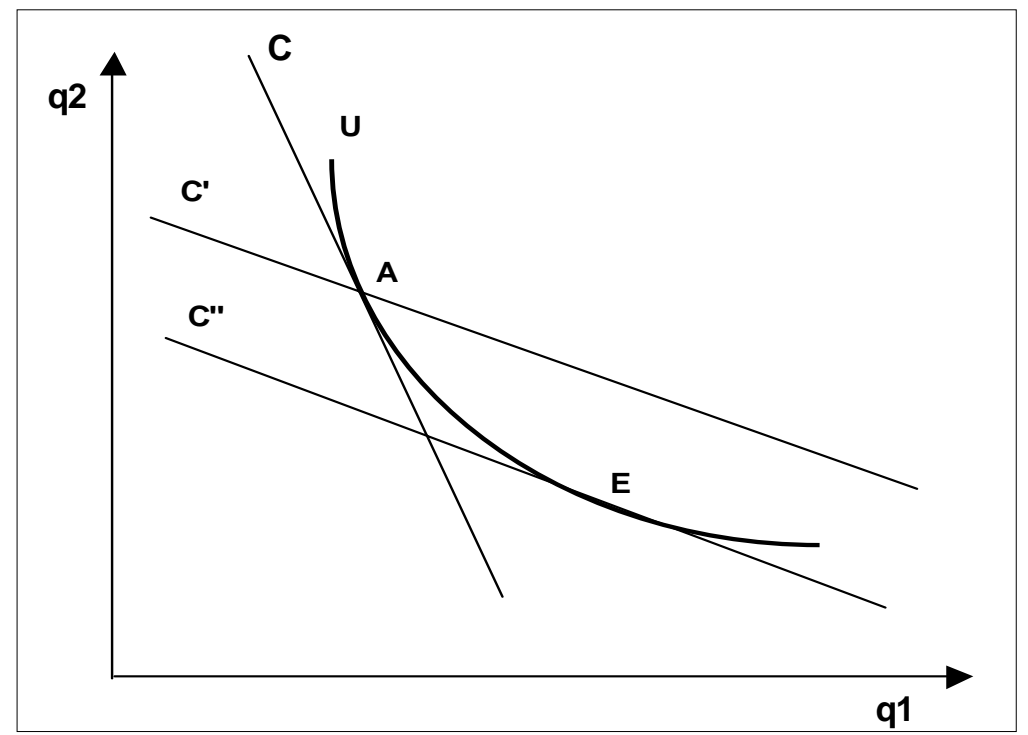

Fuente: Elaboración propia.

dades tendrá un costo igual a $C^{\prime}$. Sin embargo, si sustituye ambos bienes entre sí, alcanzará el equilibrio en el punto E, con el mismo nivel de utilidad pero a un menor costo $\left(C^{\prime \prime}\right)$.

En términos de la gráfica 2, un índice de Laspeyres representa la situación en la cual el consumidor no cambia la combinación de las cantidades consumidas; mientras tanto, un índice de costo de vida debería reflejar la situación del nuevo equilibrio. En la medida en que el primero se encuentra más alejado del origen que el segundo, sobrestima la verdadera evolución del costo de vida.

En la literatura referida al tema se han propuesto diferentes formas de aproximar los índices de costo de vida intentando superar las limitaciones de los índices tipo Laspeyres. Una aproximación ha sido la de establecer los límites inferiores y superiores de la evolución del costo de vida. ${ }^{6}$ Otra fue establecer estimaciones directas del costo

${ }^{6}$ Paasche es un límite inferior y Laspeyres es un límite superior. 
"verdadero", para lo que se han propuesto diversas fórmulas: Fisher, Tornqvist y CES.

Concretamente, un índice de costo de vida "verdadero" se define como la ratio de los mínimos costos de alcanzar un determinado nivel de vida en dos situaciones de precios. Dentro de este tipo se encuentra el índice CES, el cual tiene algunas propiedades que lo hacen atractivo para este trabajo.

\section{El índice de costo de vida CES}

Dentro de los denominados índices de costo de vida "verdaderos", aquellos que se derivan de una definición previa de la función de utilidad del consumidor son llamados "exactos". En el caso CES se supone que el consumidor tiene una función de utilidad del tipo:

$$
U=\left[\sum_{i=1}^{n} \alpha_{i} q_{i}^{\rho}\right]^{1 / \rho} \quad \alpha_{i}>0, \rho<1
$$

donde $q_{1}$ son las cantidades consumidas de cada bien. Esta función tiene la propiedad de que la elasticidad de sustitución entre cualquier par de bienes es constante e idéntica $(\sigma)$.

Es posible demostrar que el índice de costo de vida para este consumidor es del tipo:

$$
T=\left[\sum_{i=1}^{n} a_{i}^{o}\left(p_{i}^{1} / p_{i}^{0}\right)^{1-\sigma}\right]^{1 /(1-\sigma)} \quad \sigma=1 /(1-\rho)
$$

donde $p_{i}$ son los precios de cada bien en el período base (0) y en el corriente (1), y son las participaciones de cada bien en el gasto total durante el periodo base.

La fórmula CES de evolución del costo de vida tiene tres características que la hacen atractiva. La primera es que el índice queda 
parametrizado en el valor de la elasticidad de sustitución entre bienes, lo que permite realizar un análisis de sensiblidad. En particular, si la elasticidad adopta el valor cero el índice reproduce la fórmula de Laspeyres; si tiende a uno, la función de utilidad se vuelve del tipo Cobb-Douglas y el índice de costo de vida resulta ser una media geométrica cuyos exponentes son las participaciones de cada bien en el gasto total.

La segunda característica es que la fórmula CES no requiere de conocer las cantidades consumidas en el periodo corriente. Esto es una ventaja, en la medida en que esta información, generalmente, no se encuentra disponible. Éste es el caso de Uruguay, donde no se realizan encuestas continuas de consumo.

En tercer lugar, el índice de costo de vida CES, a partir de una generalización desarrollada por Lloyd (1975), admite diferentes niveles de sustitución. Esto es, el consumidor representativo puede sustituir bienes entre sí, pero a la vez sustituir la zona geográfica donde los adquiere.

\section{El índice de costo de vida CES de un consumidor fronterizo}

El índice de costo de vida CES en varios niveles se puede adaptar a la situación de un consumidor fronterizo. Para efectos de este trabajo, se supuso que éste tiene tres niveles de sustitución. Un primer nivel corresponde al margen de la frontera donde adquiere el bien: Uruguay o Brasil; un segundo nivel, a la sustitución de artículos dentro de determinados grupos: carnes, panificados, dulces, bebidas alcohólicas, etc.; un tercer nivel refiere a la sustitución de estos grupos entre sí.

Naturalmente, el consumidor no fronterizo tiene únicamente dos niveles de sustitución: entre bienes y entre grupos de bienes.

La apertura de un tercer nivel de sustitución, correspondiente a grupos de bienes, se realizó a los efectos de suponer una elasticidad nula entre los mismos. Esto parece más realista que imponer una elasticidad de sustitución igual entre todos los bienes. 


\section{Información utilizada en las estimaciones}

Con base en el marco teórico antes definido, se calculó un índice de costo de vida para el consumidor que reside en la frontera. Posteriormente, se elaboraron los índices para consumidores que radican en el interior del país y en la capital, respectivamente.

El cálculo de los índices de costo de vida requiere definir las ponderaciones, los índices de precios, las elasticidades y el periodo base a utilizar. En esta sección se detallan las fuentes de información utilizadas y los parámetros adoptados en cada caso.

\section{La canasta de bienes, sus ponderaciones y agrupamientos}

Con el fin de confeccionar la canasta de alimentos y bebidas para el consumidor fronterizo se hizo necesario considerar únicamente aquellos bienes para los que se contaba con índices de precios, tanto en la margen uruguaya como en la brasileña. Esto dio como resultado que la canasta quedara integrada por 41 artículos.

Las ponderaciones de los artículos en la canasta se determinaron con base en la información proporcionada por la EGIH 1994-1995. El peso de cada uno de los 41 productos considerados se calculó de acuerdo con el gasto promedio de un hogar de Rivera.

Finalmente, se realizaron los agrupamientos de los bienes sustitutos entre sí: farináceos, carnes, conservas de carne, bebidas lácteas, grasas, frutas, verduras, dulces y bebidas alcohólicas. Un conjunto de 13 artículos se consideraron sin sustitutos.

\section{Los indices de precios}

La evolución de precios en todo el territorio uruguayo se siguió mediante el Índice de Precios del Consumo de Uruguay. Éste, como fue mencionado, se releva solamente en la capital del país. Sin embargo, en la medida en que Uruguay es una economía pequeña e integrada es razonable suponer que los precios en el interior siguen la misma evolución. Por su parte, la evolución de los precios en la 
margen brasileña de la frontera se siguió a partir del Índice de Precios al Consumo de Río Grande do Sul. ${ }^{7}$ Naturalmente, puesto que este último está expresado en la moneda brasileña, fue necesario convertirlo a moneda uruguaya utilizando el tipo de cambio nominal entre ambas monedas.

\section{Elasticidades}

Se realizaron los cálculos para diferentes valores de la elasticidad de sustitución entre bienes. Se consideraron: el valor 0, que corresponde a la situación en que el consumidor no realiza sustitución; el valor 1, que representa la situación en que el consumidor realiza sustituciones de tal manera que mantiene constante las participaciones del gasto realizado en los diferentes bienes de la canasta; y los valores 1.5 y 2, que corresponden a un mayor grado de sustitución.

Para la elasticidad de sustitución entre márgenes de la frontera se consideraron dos valores: uno e infinito. En ambos casos las participaciones del gasto realizado en cada país están predefinidas. ${ }^{8}$ En el primero las ponderaciones son iguales a $0.5 \mathrm{y}$ en el segundo el gasto se concentrará todo en el país más barato.9, 10

\section{Base de los índices}

Se estableció una misma base, tanto temporal como geográfica, para los diferentes índices de costo de vida de forma que pudieran establecerse comparaciones entre los niveles de los mismos. La base fue Rivera noviembre de $1994=100$.

${ }^{7}$ Estado brasileño limítrofe con Uruguay.

${ }^{8}$ No se cuenta con información del gasto realizado por parte de los riverenses en cada margen de la frontera.

${ }^{9}$ Se supone adicionalmente que los coeficientes $\alpha_{i}^{j}$ son iguales para ambos países.

${ }^{10}$ Se puede demostrar que las ponderaciones son iguales a:

$a_{i}=\alpha_{i}^{\sigma} p_{i}^{1-\sigma}\left[\sum_{j} \alpha_{j}^{\sigma} p_{j}^{1-\sigma}\right]^{-1}$ 
De esta manera, los diferentes índices elaborados pueden ser vistos como los correspondientes a un mismo consumidor que es trasladado a través del tiempo en Rivera, luego es ubicado en el interior y posteriormente en Montevideo, y en ambos lugares también es trasladado en el tiempo, a fin de determinar la evolución de su costo de vida.

\section{Resultados de las estimaciones}

En el cuadro 4 se presentan las estimaciones de los índices de costo de vida para el caso en que la elasticidad de sustitución entre bienes es nula. Las columnas 2 a 5 contienen los resultados para Montevideo, el interior y Rivera, respectivamente; para este último caso, hay dos estimaciones: una con elasticidad entre márgenes de la frontera $\left(\mathrm{s}_{j}\right)$ igual a la unidad y otra con elasticidad infinito. Finalmente, en las columnas 6 y 7 se presenta el "efecto frontera", calculado como

\section{Cuadro 4}

Evolución del costo de vida en alimentos y bebidas con efecto sustitución entre márgenes de la frontera Rivera noviembre 1994=100

\begin{tabular}{lcccccc}
\hline \multirow{2}{*}{ Año } & \multirow{2}{*}{ Montevideo Interior } & \multicolumn{2}{c}{ Rivera } & \multicolumn{2}{c}{ Efecto frontera } \\
\cline { 3 - 7 } & & & $\sigma \mathrm{j}=1$ & $\sigma \mathrm{j}=\infty$ & $\sigma \mathrm{j}=1$ & $\sigma \mathrm{j}=\infty$ \\
\hline 1995 & 161.9 & 154.5 & 115.8 & 110.0 & -25.0 & -28.8 \\
1996 & 207.9 & 197.0 & 144.5 & 136.1 & -26.6 & -30.9 \\
1997 & 247.1 & 233.8 & 168.8 & 156.6 & -27.8 & -33.0 \\
1998 & 266.5 & 252.6 & 182.5 & 167.8 & -27.8 & -33.6 \\
1999 & 274.2 & 259.0 & 160.0 & 126.9 & -38.2 & -51.0 \\
2000 & 293.7 & 277.2 & 178.2 & 146.6 & -35.7 & -47.1 \\
2001 & 303.4 & 285.9 & 174.2 & 136.2 & -39.1 & -52.4 \\
2002 & 357.8 & 337.3 & 219.7 & 184.2 & -34.9 & -45.4 \\
2003 & 444.4 & 421.3 & 301.3 & 272.7 & -28.5 & -35.3 \\
2004 & 490.8 & 465.9 & 329.8 & 293.8 & -29.2 & -36.9 \\
$2005^{\text {a }}$ & 498.8 & 472.6 & 327.0 & 286.0 & -30.8 & -39.5 \\
& & & & & & \\
\hline a Primer & & & & & & \\
\end{tabular}

a Primer cuatrimestre. 
la diferencia porcentual entre el costo de vida en Rivera y el correspondiente al interior.

Durante todo el periodo analizado se observa el mismo ordenamiento; de mayor a menor costo de vida se encuentran Montevideo, el interior, Rivera con elasticidad unitaria y Rivera con elasticidad infinito.

Este ordenamiento, en cierta forma, resulta obvio; por un lado, porque Montevideo y el interior recogen la misma evolución de precios y su única diferencia está en el nivel de partida; por otro, el costo de vida en Rivera con elasticidad infinito es necesariamente inferior al correspondiente al interior porque en aquella ciudad el consumidor compra al menor precio entre el interior y la margen brasileña. ${ }^{11}$

Sin embargo, lo que el cuadro permite visualizar es la magnitud y la evolución del denominado "efecto frontera". En primer lugar, corresponde destacar la significación del mismo. Durante el periodo analizado, el consumidor fronterizo que adquirió cada bien en la margen de menor precio tuvo un costo de vida, en promedio, $39.4 \%$ inferior al que hubiera tenido en el interior del país. Esta diferencia como mínimo fue de $28.8 \%$ y como máximo llegó a alcanzar un 52.4 por ciento.

En segundo lugar, se observa que la evolución del "efecto frontera" se encuentra estrechamente vinculada a las fluctuaciones del tipo de cambio real bilateral (gráficas 1 y 3). Tal es así que aquél presenta dos cambios de nivel significativos. El primero es coincidente con la devaluación de la moneda brasileña de 1999 e implicó un aumento de 10.5 y 17.5 puntos porcentuales para los casos con elasticidad unitaria e infinito, respectivamente. El segundo es provocado por la devaluación de la moneda uruguaya de julio de 2002 y se reflejó en un salto de similar magnitud que el anterior (10.6 y 17.1, respectivamente), pero en sentido contrario. A diferencia del anterior, en este caso el impacto se recogió en dos años: 2002 y 2003.

Por su parte, en el cuadro 5 se presentan las estimaciones de los índices de costo de vida para el caso en que, además de la sustitución

11 El ordenamiento entre el costo de vida en Rivera con elasticidad unitaria y el resto de los índices no resulta obvio. 


\section{Gráfica 3}

Efecto frontera: diferencia de costo de vida entre Rivera y el interior del país

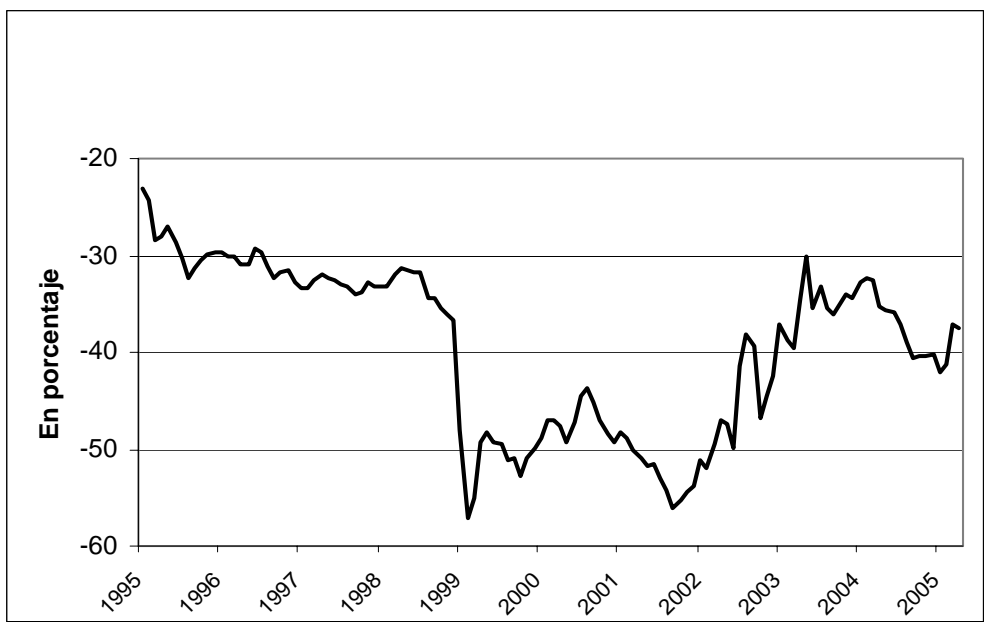

entre márgenes, existe sustitución entre bienes. El cuadro tiene la misma estructura que el anterior pero es más extenso porque contiene estimaciones para tres valores de la elasticidad de sustitución entre bienes: $\sigma=1 ; \sigma=1,5$ y $\sigma=2$.

El análisis de estos resultados permite arribar a dos nuevas constataciones. La primera es que, durante el periodo analizado, fue mucho más importante en la determinación del costo de vida de un consumidor contar con la posibilidad de sustituir bienes entre ambas márgenes de la frontera, que su mayor o menor propensión a sustituir diferentes bienes entre sí. En efecto, mientras el "efecto frontera" en promedio es superior al 30\%, la reducción en el costo de vida atribuible a la mayor propensión a sustituir bienes entre sí es como máximo 3 por ciento.

Cabe señalar que este resultado puede estar influido por la elección de la canasta y, sobre todo, por la conformación de los grupos de bienes sustitutos entre sí. Sin embargo, la diferencia entre las dos magnitudes mencionadas resulta ser muy elocuente.

La segunda constatación es que el "efecto frontera" es decreciente con la elasticidad de sustitución entre bienes. Este resultado parece 
intuitivamente razonable: el consumidor que le saca más provecho a la posibilidad de comprar en ambas márgenes de la frontera es aquel que no ajusta las cantidades compradas de cada bien a las variaciones relativas de los precios. De esta forma, encuentra toda su protección comprando fuera del país. Por el contrario, el consumidor que se protege en dos niveles, sustituyendo bienes y lugar (país) de compra, depende menos de este último.

De lo anterior se desprende que el consumidor más perjudicado por una política de "cero kilo", por la cual no se permita comprar en la margen brasileña, es aquel que no sustituye bienes en su canasta. Bajo dicha hipótesis, compraría las mismas cantidades de cada bien que adquiere actualmente pero a un precio igual o mayor; por el contrario, el consumidor que tiene una mayor elasticidad de sustitución entre bienes no sería tan perjudicado, porque se protegería reduciendo las compras de los bienes más caros internamente.

Respecto a este segundo resultado, cabe puntualizar que si bien la magnitud del "efecto frontera" es sensible a la elección del parámetro correspondiente a la elasticidad de sustitución entre bienes, las diferencias son muy pequeñas.

\section{Conclusiones}

El fenómeno de la compra de bienes y servicios en la margen brasileña de la frontera, sobre todo por parte de los habitantes de la zona, es bien conocido en el Uruguay; sin embargo, no existía hasta el momento una estimación de su impacto sobre el costo de vida de la población. En el presente trabajo, sorteando las dificultades existentes por la carencia de información, se realiza una estimación de dicho impacto.

De las estimaciones realizadas se desprende que la ventaja que tiene un consumidor por residir próximo a la frontera es significativa. En promedio, durante el periodo 1995-2005 el costo de vida en alimentos y bebidas en la frontera fue un $39.4 \%$ inferior al correspondiente al interior del país.

Esta diferencia de costo de vida experimentó fuertes fluctuaciones básicamente determinadas por inestabilidad del tipo de cambio real bilateral. Es así que luego de la devaluación de la moneda brasileña 


\section{Cuadro 5}

Evolución del costo de vida en alimentos y bebidas con efecto sustitución entre bienes y entre márgenes de la frontera Rivera noviembre $1994=100$

\begin{tabular}{lcccccr}
\hline \multirow{2}{*}{ Año } & Montevideo & Interior & \multicolumn{2}{c}{ Rivera } & \multicolumn{2}{c}{ Efecto frontera } \\
\cline { 4 - 7 } & & & $\sigma j=1$ & $\sigma j=\infty$ & $\sigma j=1$ & $\sigma j=\infty$ \\
\hline 1995 & 158,8 & 151,7 & 115,0 & 108,9 & $-24,2$ & $-28,2$ \\
1996 & 204,8 & 194,3 & 144,0 & 135,3 & $-25,9$ & $-30,4$ \\
1997 & 243,2 & 230,7 & 167,8 & 155,6 & $-27,3$ & $-32,6$ \\
1998 & 263,4 & 250,3 & 181,7 & 167,0 & $-27,4$ & $-33,3$ \\
1999 & 270,5 & 256,5 & 159,2 & 126,2 & $-37,9$ & $-50,8$ \\
2000 & 287,7 & 272,8 & 177,2 & 145,7 & $-35,0$ & $-46,6$ \\
2001 & 298,5 & 282,5 & 173,3 & 135,4 & $-38,7$ & $-52,1$ \\
2002 & 352,2 & 333,0 & 218,6 & 183,4 & $-34,3$ & $-44,9$ \\
2003 & 439,3 & 417,2 & 300,0 & 271,1 & $-28,1$ & $-35,0$ \\
2004 & 485,6 & 462,4 & 328,3 & 292,0 & $-29,0$ & $-36,8$ \\
$2005^{a}$ & 491,1 & 467,2 & 324,9 & 284,2 & $-30,5$ & $-39,2$ \\
\hline & & & $\sigma=1,5$ & & & \\
\hline 1995 & 157,3 & 150,4 & 114,6 & 108,5 & $-23,8$ & $-27,9$ \\
1996 & 203,4 & 193,1 & 143,8 & 135,0 & $-25,5$ & $-30,1$ \\
1997 & 241,3 & 229,3 & 167,3 & 155,1 & $-27,0$ & $-32,3$ \\
1998 & 261,8 & 249,1 & 181,4 & 166,6 & $-27,2$ & $-33,1$ \\
1999 & 268,7 & 255,2 & 158,9 & 125,9 & $-37,7$ & $-50,6$ \\
2000 & 284,9 & 270,7 & 176,8 & 145,2 & $-34,7$ & $-46,4$ \\
2001 & 296,1 & 280,8 & 172,9 & 134,9 & $-38,4$ & $-51,9$ \\
2002 & 349,3 & 330,9 & 218,1 & 183,0 & $-34,1$ & $-44,7$ \\
2003 & 436,9 & 415,2 & 299,4 & 270,3 & $-27,9$ & $-34,9$ \\
2004 & 483,0 & 460,6 & 327,5 & 291,2 & $-28,9$ & $-36,8$ \\
$2005^{a}$ & 487,4 & 464,6 & 323,9 & 283,4 & $-30,3$ & $-39,0$ \\
\hline & & & $\sigma=2$ & & & \\
\hline 1995 & 156,0 & 149,3 & 114,3 & 108,1 & $-23,5$ & $-27,6$ \\
1996 & 202,0 & 191,9 & 143,5 & 134,7 & $-25,2$ & $-29,8$ \\
1997 & 239,5 & 227,9 & 166,9 & 154,7 & $-26,8$ & $-32,1$ \\
1998 & 260,2 & 248,0 & 181,0 & 166,3 & $-27,0$ & $-33,0$ \\
1999 & 267,0 & 254,0 & 158,5 & 125,7 & $-37,6$ & $-50,5$ \\
2000 & 282,2 & 268,7 & 176,4 & 144,8 & $-34,4$ & $-46,1$ \\
2001 & 293,6 & 279,1 & 172,4 & 134,5 & $-38,2$ & $-51,8$ \\
2002 & 346,6 & 328,8 & 217,5 & 182,7 & $-33,8$ & $-44,4$ \\
2003 & 434,6 & 413,4 & 298,8 & 269,6 & $-27,7$ & $-34,8$ \\
2004 & 480,6 & 459,0 & 326,8 & 290,4 & $-28,8$ & $-36,7$ \\
& 484,0 & 462,1 & 322,9 & 282,6 & $-30,1$ & $-38,9$ \\
\hline & & & & & &
\end{tabular}

${ }^{\text {a }}$ Primer cuatrimestre. 
de 1999 llegó a alcanzar un 52.4\% y se redujo con posterioridad a la devaluación uruguaya de 2002 a un 35.3 por ciento.

Adicionalmente, cabe destacar que, como se desprende de los resultados, la correcta elección del lugar de compra -Uruguay o Brasilpor parte del consumidor fronterizo es mucho más importante en la determinación de su costo de vida, que su mayor o menor propensión a sustituir bienes entre sí.

Finalmente, cabe señalar que un control aduanero más estricto sobre el ingreso de mercancías provenientes del Brasil tendría un impacto sobre los consumidores. Si bien la magnitud del mismo dependería del nivel en que se encuentre el tipo de cambio real bilateral al momento de instaurar los controles, el incremento en el costo de vida para los consumidores sería, en general, significativo.

\section{Bibliografía}

Campbell, J. y B. Lapham (2001), Real Exchange Fluctuations and the Dynamics of Retail Trade Industries on the U.S.-Canada Border, NBER Working Paper Series, 8558.

Diewert, W.E. (1995), Axiomatic and Economic Approaches to Elementary Price Indexes, NBER Working Paper Series, 5104.

(1988), The Early History of Price Index Research, NBER Working Paper Series, 2713.

Engel, C. y J. Rogers (1994), How Wide is the Border?, NBER Working Paper Series, 4829.

IBGE (2004), Indicadores IBGE. Sistema nacional de índices de preços ao consumidor INPC IPCA, Río de Janeiro.

INE (1997), Metodología. Índice de los Precios del Consumo Base Marzo 1997, Montevideo.

(1995), Encuesta de Gastos e Ingresos de los Hogares 1994-1995, Montevideo.

Lloyd, P.J. (1975), "Substitution Effects and Biases in Nontrue Price Indices", American Economic Review, 65(3), pp. 301-313.

Moulton, B. (1996), Bias in the Consumer Price Index: What the Evidence?, Bureau of Labor Statistics Working Paper 294. 
Shapiro, M. y D. Wilcox (1997), Alternative Strategies for Aggregating Prices in the CPI, NBER Working Paper Series, 5980. Varian (1992), Análisis microeconómico, Antoni Bosch, Barcelona.

Waehrer, K. (2000), A Comparison of the Substitution Effects for Input and Output Price Indexes, Bureau of Labor Statistics Working Paper 327. 